\title{
Some nematodes of fishes from central China, with the redescription of Procamallanus (Spirocamallanus) fulvidraconis (Camallanidae)
}

\author{
František Moravec ${ }^{1}$, Pin Nie ${ }^{2}$ and Guitang Wang ${ }^{2}$ \\ ${ }^{1}$ Institute of Parasitology, Academy of Sciences of the Czech Republic, Branišovská 31, 37005 České Budějovice, Czech \\ Republic; \\ ${ }^{2}$ State Key Laboratory of Freshwater Ecology and Biotechnology, and Laboratory of Fish Diseases, Institute of Hydrobiology, \\ Chinese Academy of Sciences, Wuhan 430072, Hubei Province, P. R. China
}

Key words: parasitic nematodes, Procamallanus, Camallanus, Dentiphilometra, Pingus, Eustrongylides, Proleptinae, freshwater fishes, Hubei Province, China

\begin{abstract}
The present paper comprises a systematic survey of nematodes based on helminthological examinations of 176 specimens of freshwater fishes, belonging to 22 species, from central China (mostly lakes in Hubei Province) collected during the autumn of 2001. The following six species were recorded: Procamallanus (Spirocamallanus) fulvidraconis Li, 1935, Camallanus cotti Fujita, 1927, Dentiphilometra monopteri Moravec et Wang, 2002, Pingus sinensis Hsü, 1933, Proleptinae gen. sp. larv., and Eustrongylides sp. larv. Data on their morphology, morphological variability, host range, prevalence, intensity and distribution are provided. SEM studies of $P$. fulvidraconis and larval Physalopterinae, used for the first time in these species, revealed some additional morphological details and made it possible to redescribe the former. In contrast to the existing description of $P$. fulvidraconis, this species was found to possess two spicules and a V-shaped gubernaculum with unequal arms (originally mistaken for the left spicule), as well as deirids, whose location can be considered an important taxonomic feature. Larvae of the Physalopterinae have not previously been reported from fishes in China. The finding of larval Eustrongylides in Paramisgurnus dabryanus represents a new host record. All but one nematode species from this zoogeographically interesting region are briefly described and illustrated.
\end{abstract}

From the zoogeographical point of view, the territory of China, noted for the varied types of waters and the wealth of fish species, is an interesting area where the Palaearctic and Oriental faunas come into contact. Although many papers dealing with parasites of Chinese freshwater fishes have already been published (mostly in Chinese), the present knowledge of the parasite fauna of fishes in this country still remains little known.

This paper is a continuation of the authors' earlier work (Moravec et al. 2003) presenting the results of the systematic evaluation of fish helminths collected by the Chinese-Czech research team in central China in the autumn of 2001. Whereas the first paper (Moravec et al. 2003) treated the trematodes and cestodes, the present paper deals with fish nematodes. Partial data based on the same material have been published by Moravec and Nie (2002) and Moravec and Wang (2002).

\section{MATERIALS AND METHODS}

A survey of the fish hosts examined and their localities have already been given in the paper by Moravec et al. (2003); the coordinates of the two main localities from where the fish nematodes reported in this paper were collected are as follows: Bao'an Lake: $30^{\circ} 12^{\prime}-30^{\circ} 18^{\prime} \mathrm{N}, 114^{\circ} 39^{\prime}-114^{\circ} 46^{\prime} \mathrm{E}$; Liangzi Lake: $30^{\circ} 05^{\prime}-30^{\circ} 18^{\prime} \mathrm{N}, 114^{\circ} 21^{\prime}-114^{\circ} 39^{\prime} \mathrm{E}$. The recovered nematodes were washed in physiological saline and then fixed in hot $4 \%$ formaldehyde. For light microscopical examination, the nematodes were cleared with glycerine. Drawings were made with the aid of a Zeiss drawing attachment. Specimens used for scanning electron microscopy (SEM) were postfixed in $1 \%$ osmium tetroxide, dehydrated through a graded ethanol series, critical point dried and sputter-coated with gold; they were examined using a JEOL JSM-6300 scanning electron microscope at an accelerating voltage of $15 \mathrm{kV}$. The specimens have been deposited in the Helminthological Collection of the Institute of Parasitology, Academy of Sciences of the Czech Republic, in České Budějovice (coll. nos.: P. fulvidraconis - N-199; C. cotti - N-11; D. monopteri - N-787; P. sinensis - N-114; Eustrongylides sp. - N-76) and in the Institute of Hydrobiology, Chinese Academy of Sciences, in Wuhan. Type specimens of $P$. fulvidraconis and $C$. cotti could not be located for examination and they apparently were not established and deposited by the original authors (see also Moravec and Nagasawa 1989 and Rigby et al. 1997). All measurements are in micrometres unless otherwise stated. The scientific names of fishes are according to Froese and Pauly (2003).

\section{REVIEW OF SPECIES}

Family C a m a 11 a n id a e Railliet et Henry, 1915

\section{Procamallanus (Spirocamallanus) fulvidraconis} Li, 1935

Figs. 1, 2

Description. Medium-sized nematodes with thick, transversely striated cuticle. Mouth aperture oval, surrounded by six flat, crescent-shaped elevations and eight 

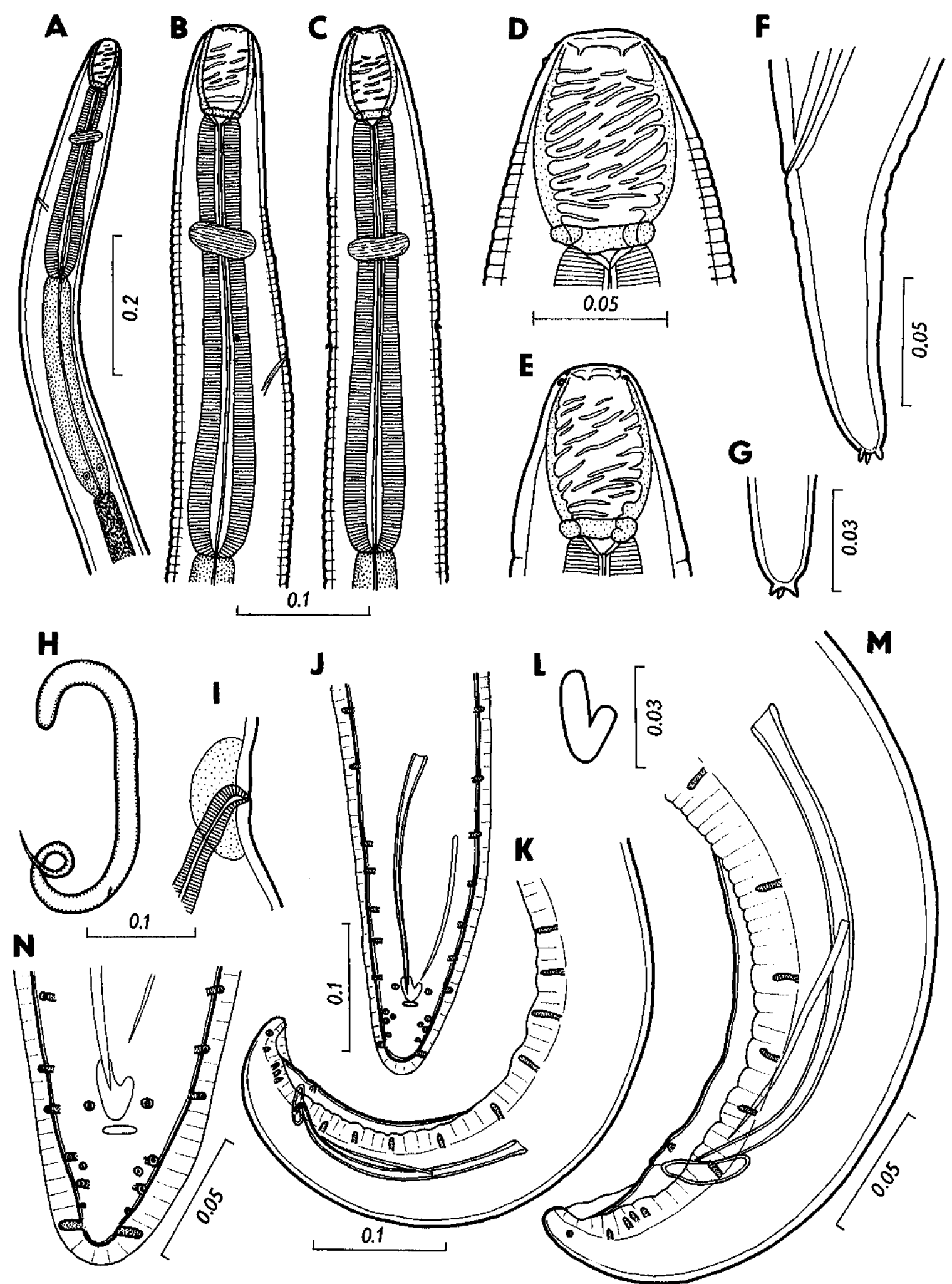

Fig. 1. Procamallanus (Spirocamallanus) fulvidraconis Li, 1935. A - anterior part of male body, lateral view; B, C - anterior end of male, lateral and dorsoventral views; $\mathbf{D}, \mathbf{E}$ - buccal capsule of gravid female and male, respectively, lateral views; $\mathbf{F}$ - female tail; $\mathbf{G}$ - tail tip of female; $\mathbf{H}$ - larva from uterus; $\mathbf{I}$ - region of vulva; $\mathbf{J}, \mathbf{K}$ - posterior end of male, ventral and lateral views; $\mathbf{L}$ gubernaculum, ventral view; $\mathbf{M}$ - posterior end of male, lateral view; $\mathbf{N}$ - tail of male, ventral view. Scale bars in $\mathrm{mm}$. 
submedian cephalic papillae arranged in two circles, each formed by four papillae; pair of small lateral amphids present. Buccal capsule yellowish, distinctly elongate, with simple, well-developed basal ring. Inner surface of whole capsule provided with numerous, interrupted spiral thickenings forming short, irregularly arranged ridges (numbering about 14-32 in lateral view). Muscular oesophagus slightly shorter than glandular oesophagus; posterior ends of both parts of oesophagus only slightly expanded. Deirids very small, simple, situated well posterior to level of nerve ring. Excretory pore approximately at mid-way between nerve ring and posterior end of muscular oesophagus. Males considerably smaller than females.

Male (7 specimens): Length of body 3,441-4,651, maximum width 72-105. Buccal capsule including basal ring 72-81 long and 42-48 wide; width/length ratio of capsule $1: 1.6-1.8$; basal ring 9 long and 30-36 wide. Length of muscular oesophagus 315-360, width 45-57; length of glandular oesophagus 336-441, width $45-60$; length ratio of muscular and glandular oesophagus $1: 1.07-1.23$. Length of entire oesophagus and buccal capsule representing $19-22 \%$ of body length. Nerve ring, deirids and excretory pore 159-174, 207 252 and 279-291, respectively, from anterior extremity. Posterior end of body ventrally bent, provided with narrow caudal alae supported by pedunculate papillae. Preanal papillae: 9-10 pairs of subventral pedunculate papillae (in one male 9 papillae on one side and 10 papillae on other side of body) and 1 pair of ventral sessile papillae situated anterior to cloacal opening; postanal papillae: 4 pairs, followed by a pair of large lateral phasmids situated near tail tip. Spicules similar in shape, unequal in length, with sharply pointed distal ends. Large (right) spicule well sclerotized, orangecoloured, 219-255 long; small (left) spicule poorly sclerotized and, therefore, hardly visible, 156-168 long; length ratio of spicules 1 : 1.38-1.67. Gubernaculum small, well sclerotized, colourless, 36-42 long in lateral view; in dorsoventral view, gubernaculum V-shaped, right arm approximately twice as long as left arm. Tail conical, 45-51 long, with rounded tip.

Female (5 gravid specimens with larvae): Length of body 9,506-13,369, maximum width 218-245. Buccal capsule including basal ring 87-90 long and 57-60 wide; width/length ratio of capsule $1: 5$; basal ring 12 long and 39-45 wide. Length of muscular oesophagus 381-490, width 69-75; length of glandular oesophagus $490-585$, width 66-69; length ratio of muscular and glandular oesophagus $1: 1.19-1.32$. Length of entire oesophagus and buccal capsule representing $9-11 \%$ of body length. Nerve ring, deirids and excretory pore 171-204, 231-286 and 354-503, respectively, from anterior extremity. Vulva distinctly postequatorial, 2,434-3,182 from posterior extremity (at 73-76\% of body length), without elevated lips. Vagina directed posteriorly from vulva. Body of larvae in uterus 15 wide. Tail conical, 111-138 long, with three small conical cuticular spikes (one dorsal and two subventral) 3-5 long.

H o s t : Pelteobagrus fulvidraco (Richardson) (Bagridae, Siluriformes).

Site of infection: Stomach.

L o c a 1 i t y: Bao'an Lake, Hubei Province, central China (27 September 2001).

Prevale nce and intensity: 66\% (25 fishes infected / 38 fishes examined); 1-36 (mean 6) nematodes per fish.

Comments. Specimens of the present material are morphologically and biometrically very similar to $P$. fulvidraconis, a species described by $\mathrm{Li}$ (1935) from the stomach of the same host species ( $P$. fulvidraco) from central China and, therefore, there is no doubt that they belong to this species. In contrast to the present study, Li (1935) did not observe the poorly sclerotized left spicule in this species and considered the gubernaculum to be the spicule; the same mistake was later followed by other authors. However, the left spicule is weakly sclerotized and hardly visible in many camallanids, in which the gubernaculum was often considered to be the left spicule (see De and Moravec 1980). The gubernaculum (reported as the left spicule) in P. fulvidraconis was originally described as heart-shaped in ventral view, but, in fact, it is V-shaped, with the right proximal arm markedly longer than the left arm; apparently, this is because the right arm supports the well-developed and well-sclerotized right spicule, whereas the opposite left arm, associated with the poorly developed left spicule, is reduced. The same shape of the gubernaculum and a poorly developed left spicule are also present, for example, in Procamallanus spiculogubernaculus Agarwal, 1958, a parasite of freshwater catfishes in India (see De and Moravec 1980).

Li (1935) reported nine pairs of preanal and five pairs of postanal subventral papillae and illustrated additional three pairs of ventral circumcloacal papillae; this study shows that the number of preanal papillae may be 9-10 pairs and, as confirmed by SEM, the postanal and ventral papillae near the cloaca are less numerous. Deirids and phasmids were not previously described in this species. The deirids of $P$. fulvidraconis are located in the region posterior to the nerve ring, in which this species differs from the majority of its congeners. Although deirids in Procamallanus spp. are not easily observable, their position appears to be one of the most reliable interspecific features in these nematodes.

Procamallanus fulvidraconis has been reported from its type host ( $P$. fulvidraco) and some other fish species (Siluridae, Cyprinidae, Channidae) from China and the Russian Far East (Li 1935, Dogel and Akhmerov 1959, Bykhovskiy 1962, Chen 1973, Wang et al. 1978, 1979, Wang 1984, Wu 1984, Wu et al. 1991). The data by Skryabina (1974) on the occurrence of this species in Central Asia proved to be erroneous (see Moravec and 

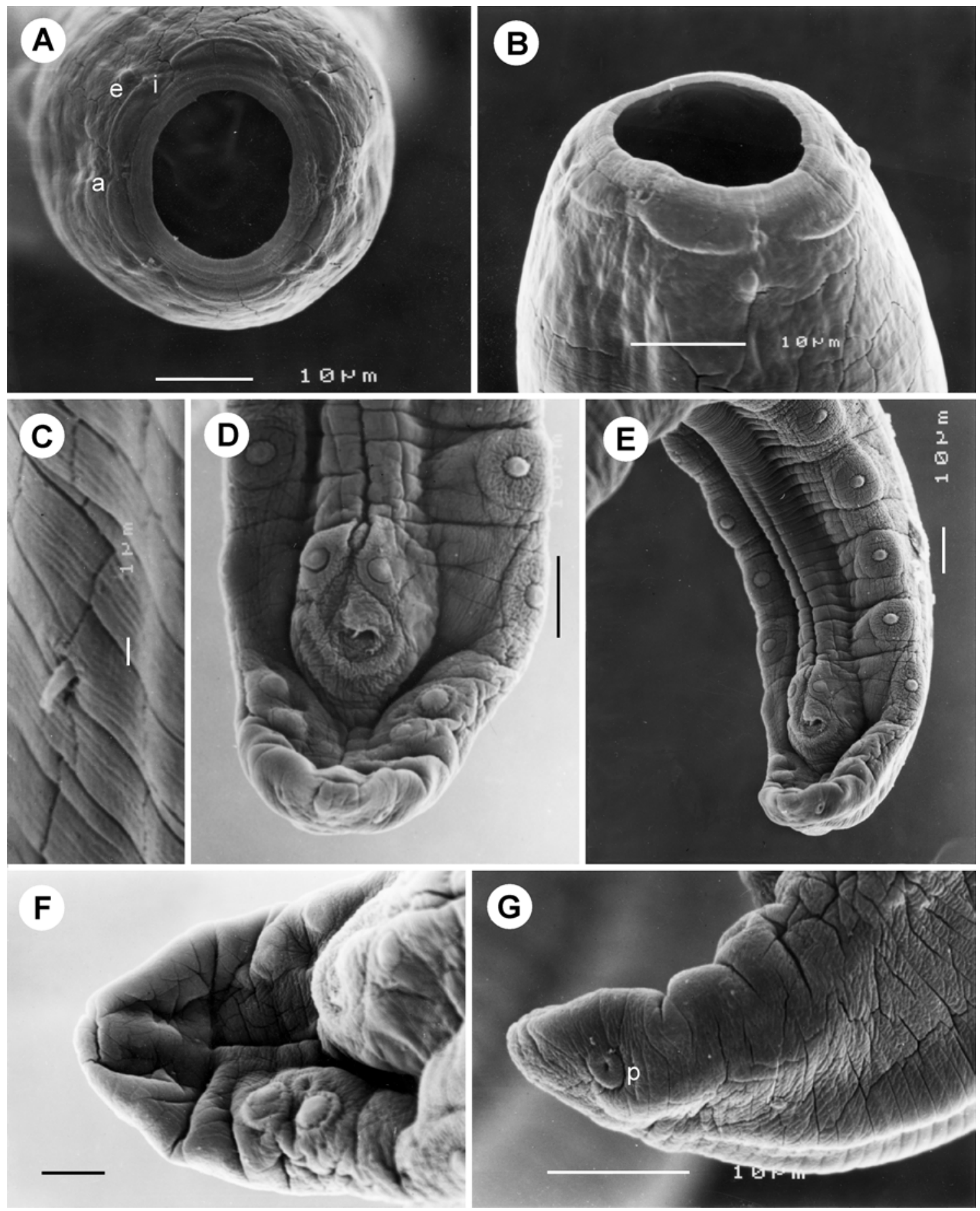

Fig. 2. Procamallanus (Spirocamallanus) fulvidraconis Li, 1935, scanning electron micrographs. A, B - cephalic end, apical and sublateral views; C - deirid; D - region of cloaca, ventral view; $\mathbf{E}$ - posterior end of male, ventral view; $\mathbf{F}, \mathbf{G}$ - tip of male tail, ventral and lateral views. $\mathrm{a}$ - amphid; $\mathrm{e}$ - external cephalic papilla; $\mathrm{i}$ - internal cephalic papilla; $\mathrm{p}$ - phasmid.

Amin 1978). Moravec and Sey (1988a) provisionally assigned the female specimens collected from two species of catfishes in Vietnam to P. fulvidraconis; however, their comparison with the present specimens from China shows that they markedly differ from $P$. fulvidraconis in the location of deirids and the extent of spiral ridges in the buccal capsule; the Vietnamese specimens probably belong to P. bagarii Karve et Naik, 1951. 
Two other similar Procamallanus spp., P. parasiluri Fujita, 1927 and P. fukiensis Wang et Ling, 1975, are reported from Silurus asotus in China. Since both are inadequately described and S. asotus is reported among the hosts of P. fulvidraconis (see Dogel and Akhmerov 1959, Chen 1973), their redescriptions are highly desirable.

The present data show a high degree of prevalence (66\%) of P. fulvidraconis in P. fulvidraco in Bao'an Lake; Wu et al. (1991) reported a prevalence $20 \%$ in the same host species from this locality.

The life cycle of $P$. fulvidraconis involves copepod intermediate hosts (Li 1935).

\section{Camallanus cotti Fujita, 1927}

Fig. 3

Syn. Camallanus zacconis Li, 1941; C. fotedari Raina et Dhar, 1972.

Description (based on specimens from $P$. fulvidraco and $S$. chuatsi; measurements of juvenile male from $C$. erythropterus in parentheses). Medium-sized nematodes with slightly transversely striated cuticle and large orange-brown capsule typical of genus. Mouth aperture slit-shaped, surrounded by four submedian oral papillae, two lateral amphids and four sclerotized plates. Valves of capsule roughly pentagonal in lateral view, internally bearing smooth longitudinal ridges, some of them incomplete. Narrow, sclerotized ring present at bottom of capsule. Tridents large, only moderately surpassing posterior border of buccal capsule. Excretory pore somewhat posterior to level of nerve ring. Deirids small, slightly asymmetrical, located at about two thirds of muscular oesophagus.

Male (3 specimens; measurements of juvenile male in parentheses): Length of body 3,577-4,406 (1,836), maximum width 136-178 (95). Buccal capsule including basal ring 96-102 (84) long, maximum width 99$105(84)$; size of basal ring $12-15 \times 57(9 \times 51)$; length of tridents 84-93 (75). Each valve of capsule strengthened internally by 16-17 (17) longitudinal ridges, 2 (2) of them being incomplete. Muscular oesophagus 348 503 (333) long, 72-87 (48) wide; glandular oesophagus 450-571 (315) long, 72-90 (42) wide; length ratio of both parts of oesophagus $1: 1.14-1.29$ (1:0.95). Nerve ring, excretory pore and deirids 186-201 (177), 195240 (183) and 207-237 (237), respectively, from anterior extremity. Posterior end of body with caudal alae. Pedunculated papillae: 7 pairs preanal and 6 pairs postanal; postanal papillae of first three pairs close together. Cloacal opening surrounded by 2 transverse sessile papillae. Spicules unequal, simple; length of larger (right) spicule 165-171 (162), of smaller (left), less sclerotized spicule 93-105 (105); length ratio of spicules $1: 1.63-1.77(1: 1.80)$. Length of tail 99-114 (90).

Female (5 specimens): Body length of specimens with larvae in uterus 7,072-8,976, maximum width 231-354. Buccal capsule including basal ring 141-159 long, maximum width 135-147; basal ring $15 \times 57-63$; length of tridents 114-126. Each valve of capsule with 15-17 ridges, 1-3 being incomplete. Muscular oesophagus 571-680 long, 111-120 wide; glandular oesophagus 748-830 long, 105-135 wide; length ratio of both parts of oesophagus $1: 1.14-1.42$. Nerve ring, excretory pore and deirids 245-300, 340-367 and 503-612, respectively, from anterior extremity. Tail very elongate, 1,074-1,469 long (14-18\% of body length), with rounded tip without any processes. Vulva postequatorial, 3,019-3,740 from posterior end of body (at $57-58 \%$ of body length), with elevated lips. Vagina muscular, directed posteriorly from vulva. Uterus extending posteriorly nearly to end of tail, filled with numerous larvae. Larvae from uterus 390-450 long and 15-18 wide.

H o s t s : Adults including females with larvae in Pelteobagrus fulvidraco (Richardson) (Bagridae, Siluriformes) and Siniperca chuatsi (Basilewsky) (Percichthyidae, Perciformes). One juvenile male in Culter erythropterus Basilewsky (Cyprinidae, Cypriniformes).

Site of infection: Intestine, mainly its posterior part.

L o c a 1 i t y : Bao'an Lake, Hubei Province (27 September 2001).

Prevalence and intensity: P. fulvidraco: $19 \%(3$ fish infected / 16 fish examined); 1 nematode per fish. $S$. chuatsi: 2 fish infected / 5 fish examined; 1 and 3 nematodes. C. erythropterus: 1 fish infected / 3 fish examined; 1 nematode.

Comments. The specimens of the present material correspond, more or less, to $C$. cotti, as it has been described by Moravec and Nagasawa (1989) from specimens from the type locality (Biwa Lake) in Japan, so that there is no doubt that they belong to this species. Recently $C$. cotti was redescribed again by Rigby et al. (1997) from specimens collected from Poecilia reticulata (Peters) introduced into Hawai'i but, in contrast to the morphology of specimens from eastern Asia, they illustrated the tridents of the buccal capsule reaching posteriorly to the level of the nerve ring and the larger spicule as the left one (generally in camallanids the large spicule is on the right side); therefore, it cannot be excluded that, in fact, they dealt with a different species.

Camallanus cotti has been reported from a number of fish species belonging to different families (e.g., Cyprinidae, Cobitidae, Bagridae, Siluridae, Poeciliidae, Odontobutidae, Percichthyidae, Channidae, Belontiidae, Gobiidae). It occurs in eastern, southeastern and southern Asia, namely in Japan, China, eastern Russia (Amur R. basin), Korea, Vietnam and India (Moravec and Nagasawa 1989); it has also been reported from fishes in Hawai'i, where it was apparently introduced along with the introduction of poeciliids (Font and Tate 1994, Font 1998). It also occurs in the cultures of aquarium fishes in Europe, North America and Australia, where this pathogenic parasite is being brought in along with imported exotic fishes. 

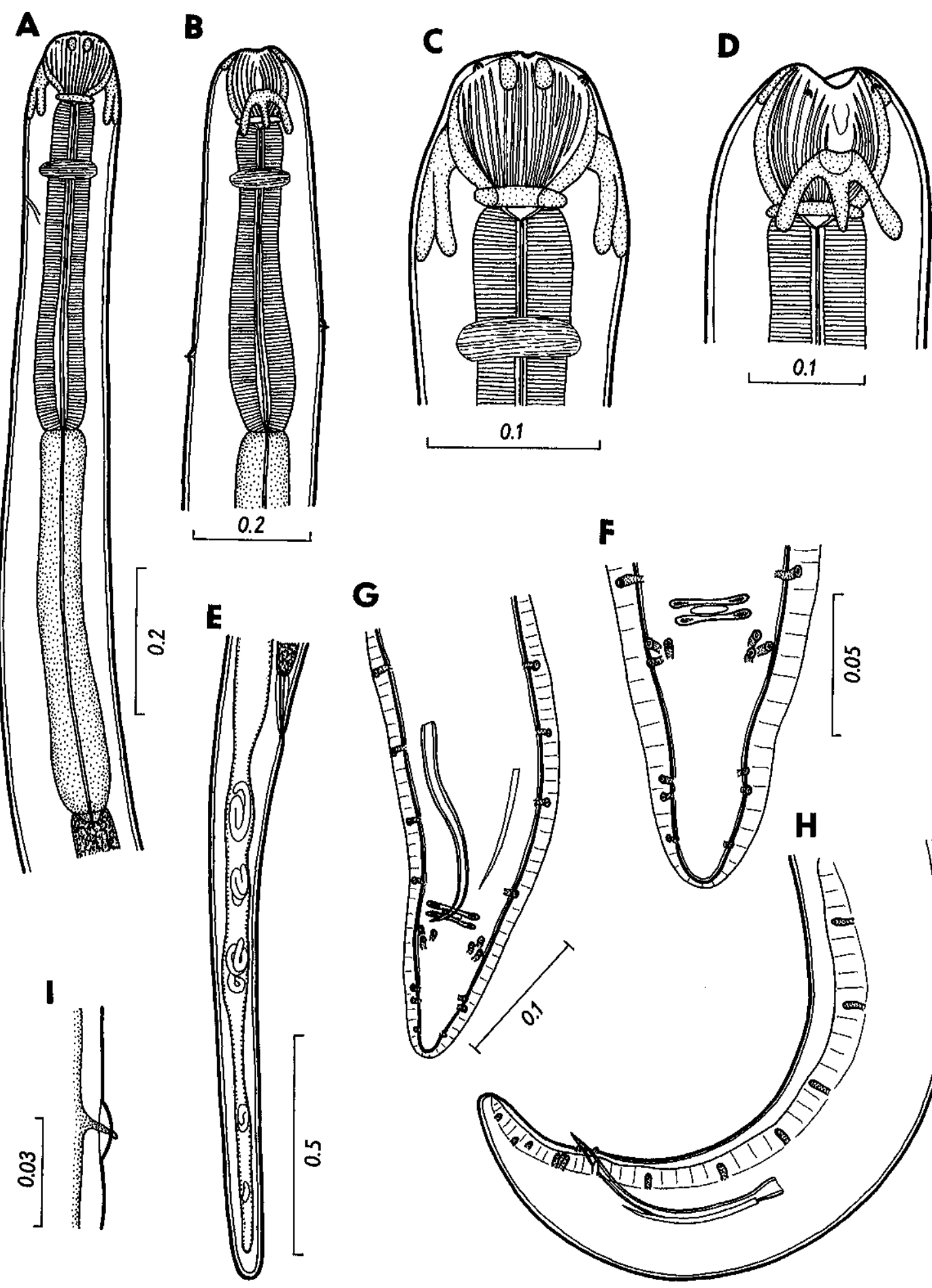

$\mathbf{G}$
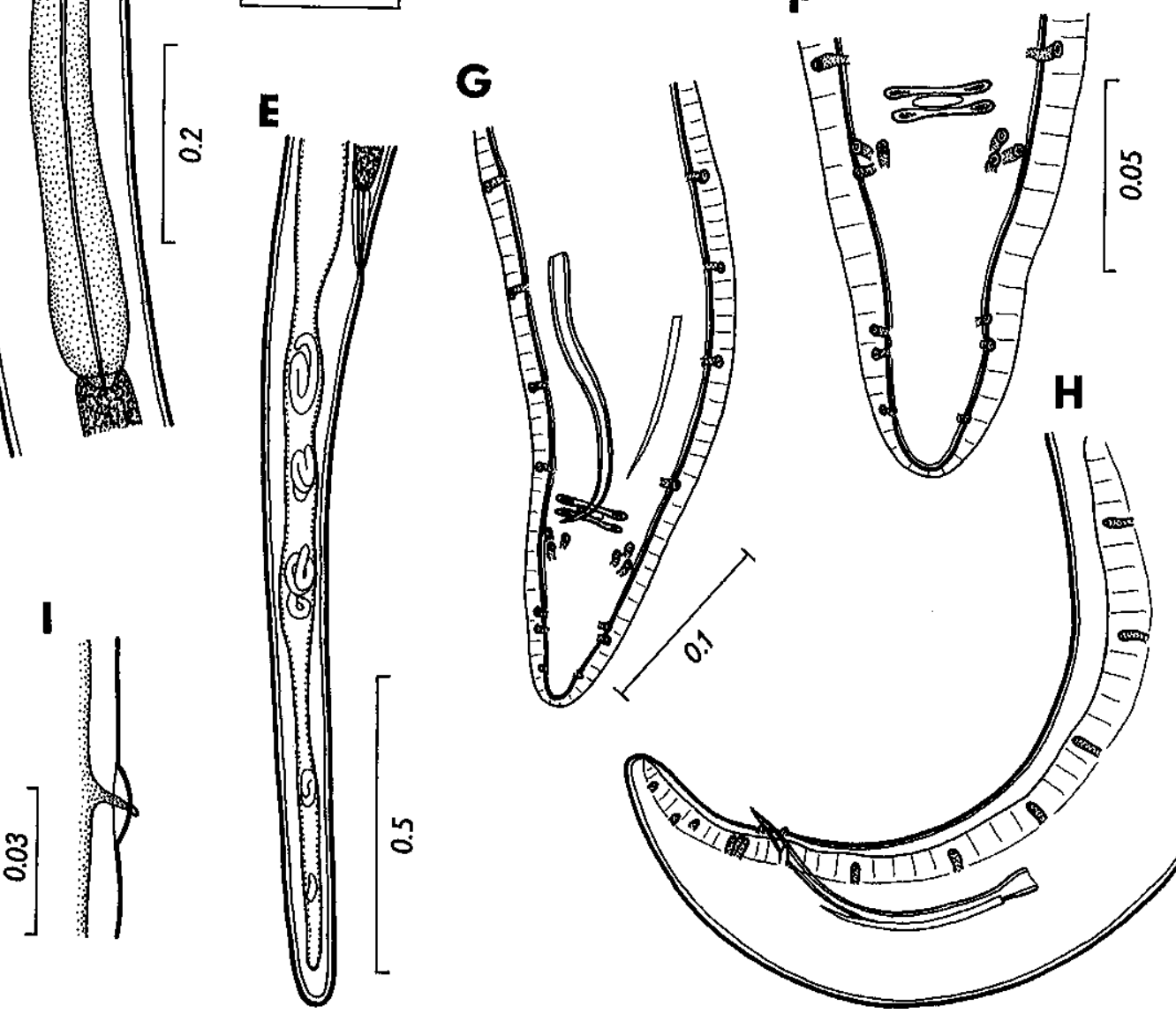

Fig. 3. Camallanus cotti Fujita, 1927. A, B - anterior end of male and female, respectively, lateral and dorsoventral views; C, D - buccal capsule of male and female, respectively, lateral and dorsoventral views; $\mathbf{E}$ - tail of gravid female, lateral view; $\mathbf{F}$ - tail of male, ventral view; $\mathbf{G}, \mathbf{H}$ - posterior end of male, ventral and lateral views; $\mathbf{I}$ - deirid, lateral view. Scale bars in mm.

In China, C. cotti has been reported by Chen (1973), Wang et al. (1979), Wang (1984), Wu (1984) and Wu et al. (1991) from many fish species including those re- corded in this study. In Bao'an Lake, it was previously recorded from seven fish species by Wu et al. (1991), of which the highest prevalence had been found in 
Odontobutis obscura (Temminck et Schlegel) (50\%); that in C. erythropterus and P. fulvidraco was $33.4 \%$ and $12.5 \%$, respectively. The present study shows a relatively high prevalence of $C$. cotti in $S$. chuatsi, which probably acquire the infection largely by feeding on other fish species harbouring this parasite.

Although many species of fish have been reported as the hosts of $C$. cotti, it is not known whether all these may serve as its true definitive hosts (i.e., in which the nematodes attain their maturity and produce larvae) or only as paratenic, paradefinitive or postcyclic hosts. The finding of only a juvenile male in $C$. erythropterus in this study suggests that small cyprinids and some other forage fishes serve mainly as the source of $C$. cotti infection for larger predatory fishes such as, for example, the Chinese perch, snakehead or catfishes.

The life cycle of $C$. cotti involves copepod intermediate hosts (Campana-Rouget et al. 1976, Levsen and Berland 2002a, b). Some authors (Stumpp 1975, Levsen 2001, Levsen and Jakobsen 2002) consider this species to be capable of a direct (monoxenous) development, but this needs a reliable verification.

Family P h i lo metrida e Baylis et Daubney, 1926

Dentiphilometra monopteri Moravec et Wang, 2002

$\mathrm{H}$ o $\mathrm{s}$ t : Monopterus albus (Zouiev) (Synbranchidae, Synbranchiformes).

Site of infection: Abdominal cavity and mesentery.

L o c a 1 i t y : Liangzi Lake, Hubei Province (29 September 2001).

Prevale n c e a d i n t e n s i t y : $25 \%$ (8 fishes infected / 32 fishes examined); 1-4 (mean 2) nematodes per fish.

Comments. This species has already been dealt with in the paper by Moravec and Wang (2002).

Family Qu i m peri i d a e Gendre, 1928

Pingus sinensis Hsü, 1933 juv.

Fig. 4 A-C

Description (1 young female). Length of body 4,338, maximum width at region of oesophagus 109. Cephalic end rounded, provided with short (15 long) vesicle, bearing four submedian papillae and lateral amphids. Cephalic end forms two lateral lobes from which broad lateral alae extend posteriorly along body; alae broadest in oesophageal region. Oesophagus undivided, 557 long (13\% of body length), slightly expanded at posterior end. Nerve ring, big deirids and excretory pore 237, 422 and 585, respectively, from anterior extremity. Vulva at posterior part of body, 1,482 from posterior extremity (at $66 \%$ of body length). Vagina directed anteriorly from vulva. Uterus without mature eggs. Tail conical, 222 long, sharply pointed.

$\mathrm{H}$ o s $\mathrm{t}$ : Channa argus (Cantor) (Channidae, Perciformes).

Site of infection: Pyloric caecum.

L o c a 1 i t y : Bao'an Lake, Hubei Province (27 September 2001).
P revale n e a nd intensity : In 1 of 6 fish examined; 1 nematode.

Comments. The identification of this specimen is based on its morphology and host type. Pingus sinensis is a specific intestinal parasite of fishes of the genus Channa. Originally it was described by Hsü (1933) from $C$. argus from China, later it was recorded a few times from the same host species from China and eastern Russia (Amur R. basin) (Wu 1956, 1984, Dogel and Akhmerov 1959, Akhmerov 1962, Belouss 1965, Chen 1973, Wang et al. 1978) and by Ha Ky (1971) and Moravec and Sey (1988c) from Channa maculata (Lacépède) from North Vietnam; the latter authors reported it also from a cyprinid fish serving probably only as an accidental host. Recently $P$. sinensis was also found in C. argus introduced into Japan (Shimazu et al. 2000). The present finding is the first report of this parasite from fishes of Bao'an Lake (cf. Wu et al. 1991).

Family Phys a lopterid a e Leiper, 1908

Proleptinae gen. sp. larva

Figs. 4 D-H, 5

Description (1 specimen). Body whitish, 8,024 long and 286 wide. Cuticle with fine transverse striations. Cephalic end rounded, with two feebly outlined lips, each of them provided with small terminal tooth, two submedian mouth papilla and lateral amphid. Oesophagus distinctly divided into short and narrow anterior muscular portion 210 long, 39 wide, and long, posteriorly expanded posterior glandular part 1,061 long, 122 wide. Nerve ring encircling muscular oesophagus at its posterior end, 218 from anterior extremity. Excretory pore and small, somewhat asymmetrical deirids situated 306 and 326/367, respectively, from anterior end. Tail conical, 190 long, with sharply pointed tip.

H o s t : Monopterus albus (Zouiev) (Synbranchidae, Synbranchiformes)

Site of infection: Mesentery.

L o c a 1 i t y : Liangzi Lake, Hubei Province (29 September 2001).

Prevalence and intensity:3\%(1 fish infected/ 32 fish examined); 1 nematode.

Comments. Since the genera of the Proleptinae differ from one another mostly in characters found only in adults (location of the vulva, type of spicules), the generic identification of this larva is impossible. Morphologically it may belong to the genera Proleptus Dujardin, 1845, Paraleptus Wu, 1927 or Heliconema Travassos, 1919; because the first two named genera include species parasitizing mainly elasmobranchs, it is highly probable that the larva belongs to the latter; freshwater species of these genera are known largely from fishes in the Oriental Region. Morphologically similar larvae encapsulated in the abdominal cavity of several species of freshwater cyprinid, siluroid and channid fishes have been reported from Afghanistan and North Vietnam by Moravec and Amin (1978) and Moravec and Sey (1988b), respectively; the former authors 


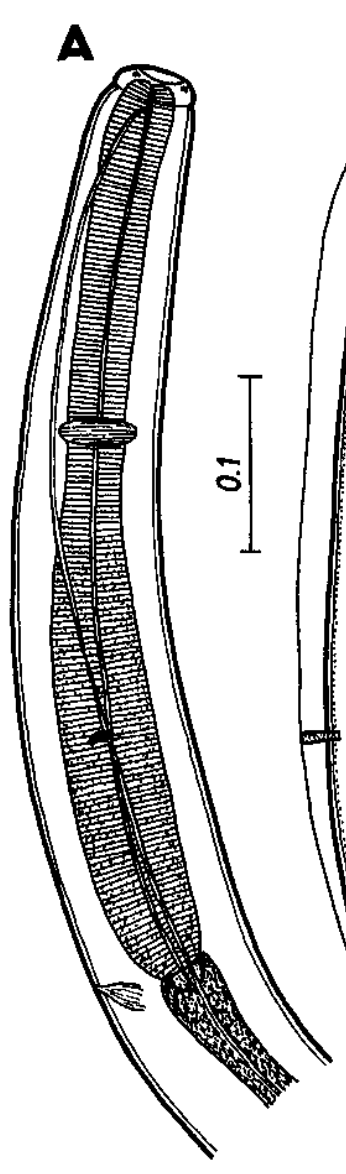

B
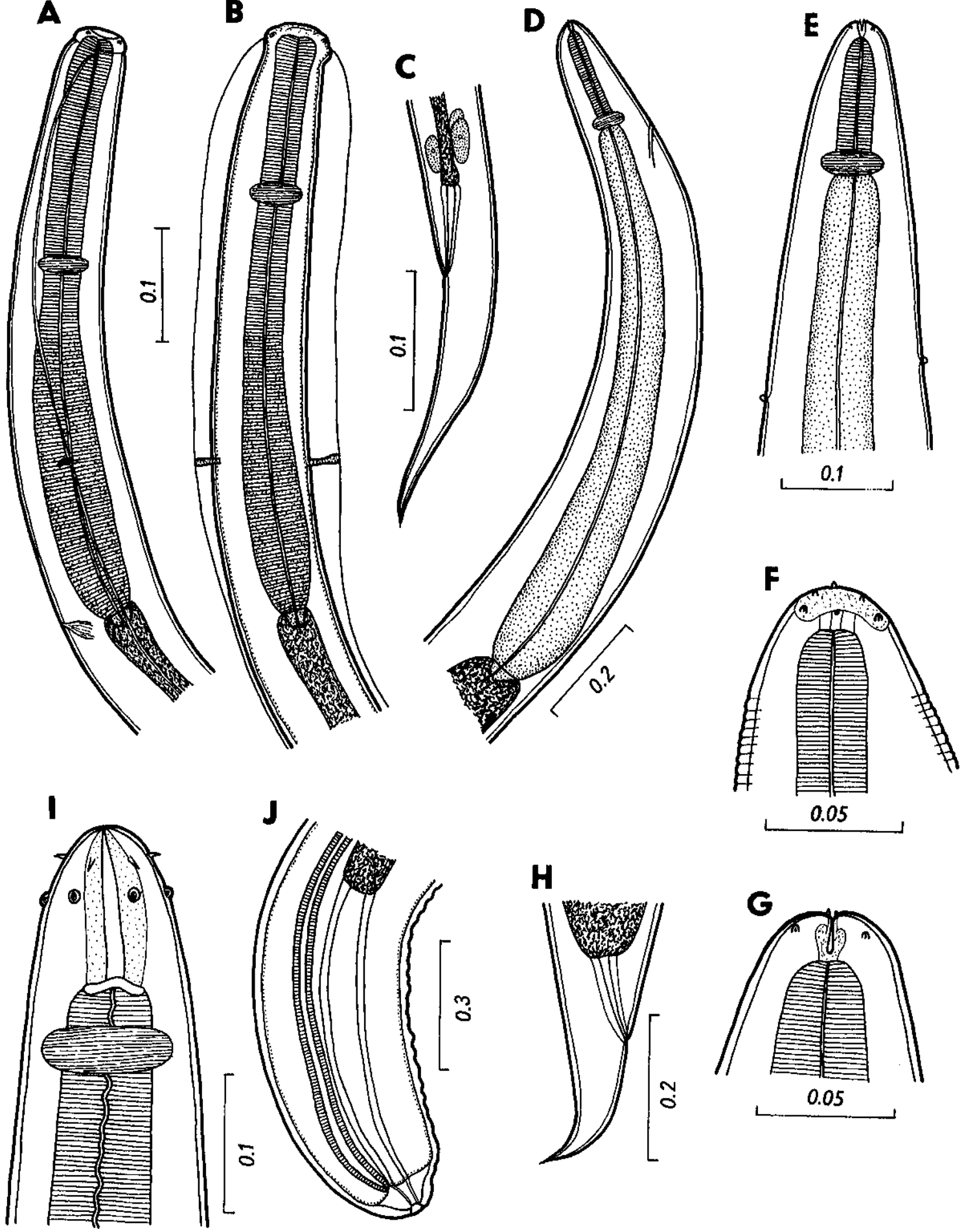
view); D-H - Proleptinae gen. sp. larva from Monopterus albus (D, E - anterior part of body, lateral and dorsoventral views; $\mathbf{F}, \mathbf{G}$ - cephalic end, lateral and dorsoventral views; $\mathbf{H}$ - tail, lateral view); I, J - Eustrongylides sp. larva from M. albus, lateral views ( $\mathbf{I}$ - cephalic end; $\mathbf{J}$ - posterior end of female larva). Scale bars in $\mathrm{mm}$.

mention that fishes harbouring these encapsulated larvae probably serve as paratenic hosts, whereas some predatory fishes (Mastacembelus, Channa) are the definitive hosts. This is the first record of these larvae from fishes in China. 

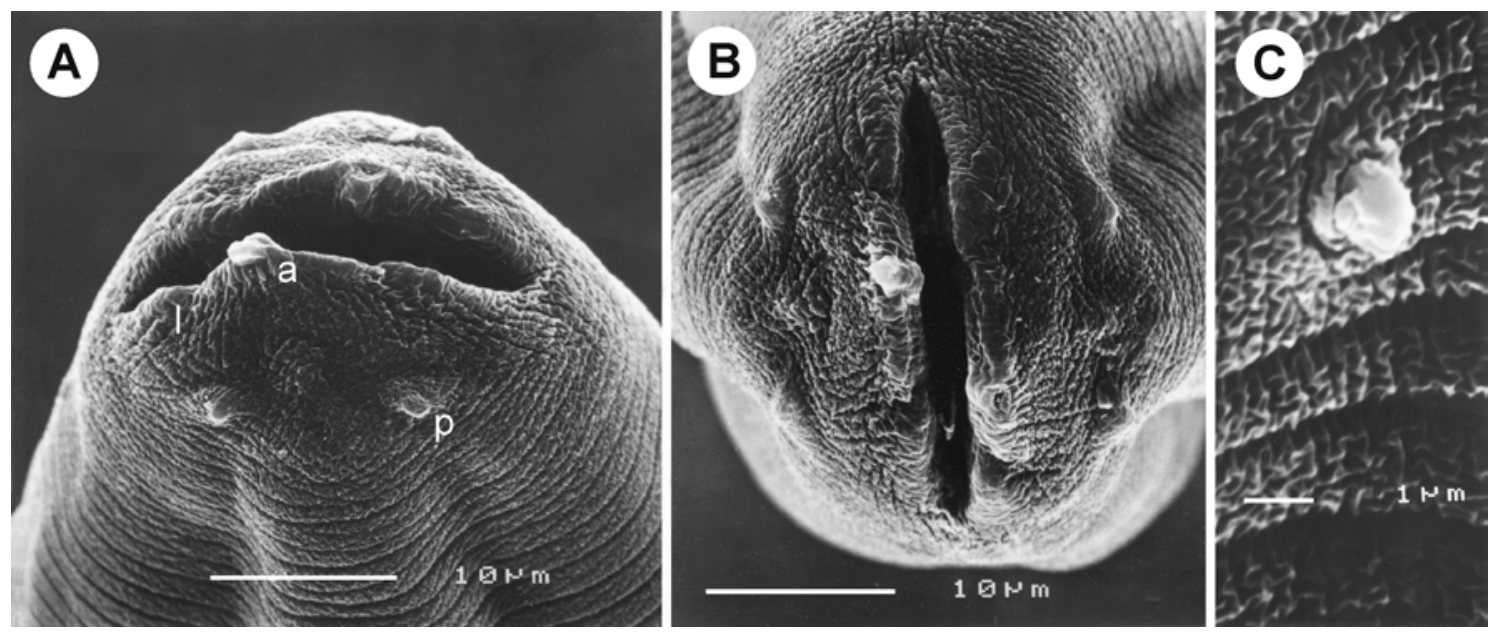

Fig. 5. Proleptinae gen. sp. larva from Monopterus albus, scanning electron micrographs. A, B - cephalic end, lateral and apical views; $\mathbf{C}$ - deirid. a - median tooth; 1 - lateral tooth; $\mathrm{p}$ - cephalic papilla.

Family Di o c to phy mat i d a e Railliet, 1915

\section{Eustrongylides sp. larva}

Fig. 4 I, J

Description (10 specimens). Body of smaller larvae whitish, of larger larvae red-brown. Length of body 32,028-50,320, maximum width 381-639. Cephalic extremity narrowed, rounded, with 12 labial papillae in two circles, each with 6 papillae. Papillae of inner circle small, spike-like, those of outer circle large, domeshaped. Buccal cavity 95-177 long. Length of oesophagus $8,704-11,628$. Nerve ring encircling oesophagus 163-258 from head end. Anus terminal and posterior extremity blunt. Female larvae with developing thickwalled vagina.

H o s t s : Paramisgurnus dabryanus Sauvage (Cobitidae, Cypriniformes) and Monopterus albus (Zouiev) (Synbranchidae, Synbranchiformes).

Site of infection: Abdominal cavity.

L o c a 1 i t i e s : Surroundings of Xinyang, Henan Province (P. dabryanus, 29 September 2001) and Liangzi Lake, Hubei Province (M. albus, 29 September 2001).

Prevalence and intensity: P. dabryanus: in 1 of 5 fish examined; 2 nematodes. M. albus: $31 \%$ (10/32); $1-$ 12 (mean 4) nematodes per fish.

Comments. The species identification of Eustrongylides larvae from fishes is problematic (unless verified by feeding experiments to bird definitive hosts), because interspecific morphological differences are found only in adults (Measures 1988). The larvae from fishes are assumed to be the third- and fourth-stage larvae and fishes may act as either the second intermediate or paratenic hosts (other paratenic hosts may be amphibians and reptiles). Data in the literature report on considerable pathogenicity of Eustrongylides larvae to host fishes, especially those heavily infected. Eustrongylides fourth-stage larvae are occasionally found as the parasites of humans after being ingested along with raw fish (e.g., Eberhard et al. 1989). The definitive hosts are various fish-eating birds.

In China, larvae of Eustrongylides were previously reported from a few fish species of the families Engraulidae, Cyprinidae, Siluridae, Bagridae, Channidae and Percichthyidae (Chen 1973, Wang et al. 1997). The present finding of these larvae in $P$. dabryanus represents a new host record.

Acknowledgements. The authors' thanks are due to Luo Haiyan of the Institute of Hydrobiology, Chinese Academy of Sciences, for her assistance in obtaining fishes, and to Iva Dyková, Institute of Parasitology, ASCR, for her help with the examination of fish. Thanks are also due to the staff of the Laboratory of Electron Microscopy of the latter institute for their technical assistance and to Irena Husáková of the Department of Helminthology from the same institute for her help with the preparation of illustrations. This study was partly supported by the grant no. ME 424 from the Ministry of Education, Youth and Sports of the Czech Republic, within the framework of the project no. 35-35 of the Cooperation in Science and Technology between the Czech Republic and People's Republic of China (programme KONTAKT), and partly by the grant no. 30025035 from the National Natural Science Foundation of China. 


\section{REFERENCES}

AKHMEROV A.Kh. 1962: [The finding of a tropical nematode of the family Pingidae Hsü, 1933 in the Amur fish.] Izv. Tikhookeans. nauch.-issled. inst. ryb. khoz. i okeanogr. 48: 212-214. (In Russian.)

BELOUSS E.V. 1965: [Nematodes of freshwater fishes from the Primorsk Territory.] In: Paraziticheskie Chervi Domashnikh i Dikikh Zhivotnykh. Vladivostok, pp. 4865. (In Russian.)

BYKHOVSKIY B.E. (Ed.) 1962: [Key to Parasites of Freshwater Fishes of the USSR.] Publ. House of the USSR Acad. Sci., Moscow and Leningrad, 776 pp. (In Russian.)

CAMPANA-ROUGET Y., PETTER A.J., KREMER M., MOLET B., MILTGEN F. 1976: Présence du nématode Camallanus fotedari dans le tube digestif de poissons d'aquarium de diverses provenances. Bull. Acad. Vét. Fr. 49: 205-210.

CHEN C. (Ed.) 1973: [An Illustrated Guide to the Fish Diseases and Causative Pathogenic Fauna and Flora in the Hubei Province.] Science Press, Beijing, 456 pp. (In Chinese.)

DE N.C., MORAVEC F. 1980: Redescription of the nematode Procamallanus spiculogubernaculus with notes on related forms. Acta Soc. Zool. Bohemoslov. 44: 81-91.

DOGEL V.A., AKHMEROV A.Kh. 1959: [Nematodes of fishes of the River Amur.] Acta Hydrobiol. Sin. 3: 272 304. (In Chinese and Russian.)

EBERHARD M.L., HURWITZ H., SUN A.M., COLETTA D. 1989: Intestinal perforation caused by larval Eustrongylides (Nematoda: Dioctophymatoidae) in New Jersey. Am. J. Trop. Med. Hyg. 40: 648-650.

FONT W.E. 1998: Parasites in paradise: patterns of helminth distribution in Hawaiian stream fishes. J. Helminthol. 72: 307-311.

FONT W.F., TATE D.C. 1994: Helminth parasites of native Hawaiian freshwater fishes: an example of extreme ecological isolation. J. Parasitol. 80: 682-688.

FROESE R., PAULY D. (Eds.) 2003: FishBase. World Wide Web electronic publication, www.fishbase.org, version 04 February 2003.

HA KY 1971: [Some species of nematodes from freshwater fishes in North Vietnam.] Parazitologiya 5: 241-250. (In Russian.)

HSÜ H.F. 1933: On some parasitic nematodes collected in China. Parasitology 24: 512-541.

LEVSEN A. 2001: Transmission ecology and larval behaviour of Camallanus cotti (Nematoda, Camallanidae) under aquarium conditions. Aquar. Sci. Conserv. 3: 315-325.

LEVSEN A., BERLAND B. 2002a: The development and morphogenesis of Camallanus cotti Fujita, 1927 (Nematoda: Camallanidae), with notes on its phylogeny and definitive host range. Syst. Parasitol. 53: 29-37.

LEVSEN A., BERLAND B. 2002b: Post-embryonic development of Camallanus cotti (Nematoda: Camallanidae), with emphasis on growth of some taxonomically important somatic characters. Folia Parasitol. 49: 231-238.

LEVSEN A., JAKOBSEN P.J. 2002: Selection pressure towards monoxeny in Camallanus cotti (Nematoda,
Camallanidae) facing an intermediate host bottleneck situation. Parasitology 124: 625-629.

LI H.C. 1935: The taxonomy and early development of Procamallanus fulvidraconis n. sp. J. Parasitol. 21: 103113.

MEASURES L.N. 1988: Revision of the genus Eustrongylides Jägerskiöld, 1909 (Nematoda: Dioctophymatoidea) of piscivorous birds. Can. J. Zool. 66: 885-895.

MORAVEC F., AMIN A. 1978: Some helminth parasites, excluding Monogenea, from fishes of Afghanistan. Acta Sci. Nat. Brno 12: 1-45.

MORAVEC F., NAGASAWA K. 1989: Observations on some nematodes parasitic in Japanese freshwater fishes. Folia Parasitol. 36: 127-141.

MORAVEC F., NIE P. 2002: Observations on two species of phylodistomes (Trematoda: Gorgoderidae) from fishes of the Hubei Province, central China, with an erection of Neophyllodistomum gen. n. Helminthologia 39: 221-225.

MORAVEC F., NIE P., SCHOLZ T., WANG G. 2003: Some trematodes and cestodes of fishes mainly from Hubei Province, central China. Acta Soc. Zool. Bohem. - in press.

MORAVEC F., SEY O. 1988a: Nematodes of freshwater fishes from North Vietnam. Part 1. Camallanoidea and Habronematoidea. Acta Soc. Zool. Bohemoslov. 52: 128148.

MORAVEC F., SEY O. 1988b: Nematodes of freshwater fishes from North Vietnam. Part 2. Thelazioidea, Physalopteroidea and Gnathostomatoidea. Acta Soc. Zool. Bohemoslov. 52: 176-191.

MORAVEC F., SEY O. 1988c: Nematodes of freshwater fishes from North Vietnam. Part 3. Coscocercoidea, Seuratoidea, Atractoidea, Heterakoidea and Ascaridoidea. Acta Soc. Zool. Bohemoslov. 52: 250-265.

MORAVEC F., WANG G.T. 2002: Dentiphilometra monopteri n. gen., n. sp. (Nematoda: Philometridae) from the abdominal cavity of the ricefield eel Monopterus albus in China. J. Parasitol. 88: 961-966.

RIGBY M.C., FONT W.F., DEARDORFF T.L. 1997: Redescription of Camallanus cotti Fujita, 1927 (Nematoda: Camallanidae) from Hawai'i. J. Parasitol. 83: 1161-1164.

SHIMAZU T., KURAMOCHI T., ARAKI J., MACHIDA M. 2000: Digenean, cestode, and nematode parasites of freshwater fishes of the Imperial Palace, Tokyo. Mem. Nat. Sci. Mus., Tokyo, No. 35, 211-231.

SKRYABINA E.S. 1974: [Helminths of Acipenserid Fishes.] Nauka, Moscow, 168 pp. (In Russian.)

STUMPP M. 1975: Untersuchungen zur Morphologie und Biologie von Camallanus cotti (Fujita, 1927). Z. Parasitenkd. 46: 277-290.

WANG P. 1984: Descriptions of three new species and a list of parasitic nematodes from vertebrates in Fujian Province. Wuyi Sci. J. 4: 113-132. (In Chinese, Engl. Summary.)

WANG P., ZHAO Y., CHEN C. 1978: On some nematodes from vertebrates in South China. Fujian Shida Xuebao, No. 2, 75-90. (In Chinese.) 
WANG P., ZHAO Y., WANG X., ZHANG J. 1979: Report on some nematodes from vertebrate animals in Central and South China. Fujian Shida Xuebao 2: 78-92. (In Chinese, Engl. summary.)

WANG W., LI L., YU Y., FENG W., XIAO C., WANG G., LIU J., YAO W., FENG S. 1997: Parasite fauna of fishes in Wuling Mountains area. In: D. Song (Ed.), Invertebrates of Wuling Mountains Area, Southwestern China. Science Press, Beijing, pp. 73-146. (In Chinese, Engl. summary.)

WU H.S. 1956: Studies on the parasitic nematodes of freshwater fishes in China 1. Acta Hydrobiol. Sin. 2: 99-106+ 3 Plts. (In Chinese, Engl. summary.)
WU H. 1984: The parasitic nematodes of fishes from Liao He in China. In: Parasitic Organisms of Freshwater Fish of China. Agricult. Publ. House, Beijing, China, pp. 177200. (In Chinese.)

WU H., LI L., FENG W., YAO W., LIU J., YU Y. 1991: A preliminary study on the fish parasitic fauna of the Bao'an Lake. In: C. Hu and X. Huang (Eds.), Collected Papers on the Fishery Ecology and Exploitation Technology of Bao'an Lake. Science Press, Beijing, pp. 160-171. (In Chinese.)

Accepted 24 February 2003 